\title{
INVESTIGACIÓN
}

Recibido: 15/04/2021 --- Aceptado: 28/10/2021 --- Publicado: 03/01/2022

\section{ANÁLISIS DE LAS COMPETENCIAS MEDIÁTICAS DEL ALUMNADO QUE INGRESA EN LA UNIVERSIDAD: UN ESTUDIO DE CASO EN ESTUDIANTES DE COMUNICACIÓN}

\author{
The analysis of the informational competencies of students entering university: \\ a case study
}

Aida María De Vicente Domínguez: Universidad de Málaga. España. aidamaria@uma.es

Mireya Rocio Carballeda Camacho: Universidad de Málaga. España. mcarballeda@uma.es

Estefania Cestino González: Universidad de Málaga. España. ecestino@uma.es

\section{Cómo citar el artículo:}

De Vicente Domínguez, A. M., Carballeda Camacho, M. R. y Cestino González, E. (2022). Análisis de las competencias mediáticas del alumnado que ingresa en la universidad: un estudio de caso en estudiantes de comunicación.Vivat Academia. Revista de Comunicación, 155, 151-171. http:/ / doi.org/10.15178/va.2022.155.e1375

\section{RESUMEN}

En la era actual es necesario formar a las nuevas generaciones en competencias que les permitan tener una alfabetización informacional. En este contexto, el objetivo general del estudio es analizar si el alumnado que llega a la Universidad tiene competencias informacionales en el acceso de la información (estrategias de búsqueda en Internet) para realizar sus trabajos académicos. Los objetivos son: identificar los recursos digitales e impresos que usan para elaborar las tareas académicas; dar a conocer si conocen metabuscadores y buscadores especializados; detectar cuál es su principal motor de búsqueda; y valorar si tienen competencias para acceder a la información mediante los indicadores booleanos, comandos y operadores. La metodología usada es un cuestionario de preguntas abiertas al alumnado, que ha ingresado en el grado de Publicidad y Relaciones Públicas de la Universidad de Málaga, en una materia obligatoria. Se concluye que ser nativos digitales no implica tener habilidades para localizar datos fiables en la ingente cantidad de información ubicada en Internet pues presentan diversas disfunciones como carecer de un conocimiento sobre los indicadores booleanos, los comandos y operadores para buscar información, y desconocen los metabuscadores y los 
De Vicente Domínguez, A. M., Carballeda Camacho, M. R. y Cestino González, E. Análisis de las competencias mediáticas del alumnado que ingresa en la universidad: un estudio de caso en estudiantes de comunicación

directorios específicos. También confunden navegador con buscador y recursos digitales con instrumentos, lo que hace preciso formales en materias específicas, cursos o de forma transversal en diversas materias. Así como, se recomienda que cada docente aporte al alumnado recursos digitales de utilidad para acceder a contenidos fiables de la materia impartida ampliando así sus habilidades para acceder a la cultura digital.

PALABRAS CLAVE: Comunicación - Alfabetización informacional - Buscadores especializados de información - Competencias informacionales -Educación Documentación en Internet.

\section{ABSTRACT}

In the current era, it is necessary to train the new generations in competencies that allow them to be information literate. In this context, the general objective of the study is to analyze whether students entering university have information literacy skills in accessing information (Internet search strategies) to carry out their academic work. The objectives are: to identify the digital and printed resources they use to prepare academic assignments; to find out if they know meta-search engines and specialized search engines; to reveal which is their main search engine; and to investigate if they have the skills to access information through Boolean indicators, commands and operators. The methodology used is a questionnaire of open questions to students who have entered the degree of Advertising and Public Relations at the University of Malaga, in a compulsory subject.Malaga, in a compulsory subject. It is concluded that being digital natives does not imply having skills to locate reliable data in the huge amount of information located on the Internet as they present various dysfunctions such as lacking knowledge about theboolean indicators, commands and operators to search for information, and lack of knowledge of meta-search engines and specific directories. They also confuse browser with search engine and digital resources with tools, which makes it necessary to have formal training in specific subjects, courses or in a transversal way in different subjects. It is also recommended that each teacher provide students with useful digital resources to access reliable content of the subject taught, thus expanding their skills to access digital culture.

KEYWORDS: Communication - Information literacy - Search engines - Skills Education - Internet. 


\section{ANÁLISE DAS COMPETÊNCIAS DE MÍDIA DE ALUNOS QUE ENTRAM NA UNIVERSIDADE: UM ESTUDO DE CASO EM ESTUDANTES DE COMUNICAÇÃO}

\section{RESUMO}

$\mathrm{Na}$ era atual, é necessário treinar as novas gerações em competências que lhes permitam ter alfabetização informacional. Nesse contexto, o objetivo geral do estudo é analisar se os alunos que chegam à Universidade possuem habilidades informacionais no acesso à informação (estratégias de busca na Internet) para a realização dos seus trabalhos acadêmicos. Os objetivos são: identificar os recursos digitais e impressos que utilizam para desenvolver trabalhos acadêmicos; mostrar se os alunos conhecem motores de meta-pesquisa e motores de pesquisa especializados; detectar qual é o seu mecanismo de pesquisa principal e avaliar se eles têm as habilidades para acessar informações por meio de indicadores, comandos e operadores booleanos. A metodologia utilizada é um questionário de perguntas abertas a alunos, que tenham ingressado no curso de Publicidade e Relações Públicas da Universidade de Málaga, em disciplina obrigatória. Conclui-se que ser nativos digitais não implica ter habilidades para localizar dados confiáveis na vasta quantidade de informações localizadas na Internet, uma vez que apresentam disfunções diversas, como falta de conhecimento de indicadores booleanos, comandos e operadores de busca de informações, e eles desconhecem os mecanismos de metabusca e diretórios específicos. Também confundem navegador com motor de busca e recursos digitais com instrumentos, o que torna necessária a formação de disciplinas específicas, cursos ou de forma transversal em várias disciplinas. Da mesma forma, recomenda-se que cada professor proporcione aos alunos recursos digitais úteis para o acesso a conteúdos confiáveis da matéria ministrada, ampliando assim suas habilidades de acesso à cultura digital.

PALAVRAS-CHAVE: Comunicação - Competência informacional - Buscadores de informação especializados - Competências informacionais - Educação Documentação na Internet.

\section{INTRODUCCIÓN}

La evolución tecnológica ha modificado las posibilidades de acceso a la información generándose una nueva cultura informativa: la digital. Sin embargo, para acceder a los datos en el soporte on line es necesario tener competencias para localizar correctamente la información buscada entre la ingente cantidad de contenidos alojados en Internet. $\mathrm{Y}$ es que si para acceder a la cultura letrada (impresa) es preciso saber leer y escribir, así como conocer dónde localizar los 
De Vicente Domínguez, A. M., Carballeda Camacho, M. R. y Cestino González, E. Análisis de las competencias mediáticas del alumnado que ingresa en la universidad: un estudio de caso en estudiantes de comunicación

recursos impresos, ahora es preciso formar al alumnado para buscar contenidos digitales (Coll, 2005).

Una competencia que forma parte de la alfabetización informacional, entendiendo por este término desde el punto de vista de las instituciones educativas y documentales, "el servicio y las actividades para lograr la enseñanza-aprendizaje de los conceptos, procedimientos y actitudes relativos al acceso y uso de la información" (Gómez Hernández, 2002, p. 5). Una formación que en la era actual se considera "un pilar en las instituciones de educación (...) para lograr que las personas se preparen y puedan desarrollar competencias informativas para cumplir sus metas tanto laborales como educativas" (Toledo y Maldonado-Radillo, 2015, p. 60).

En concreto, esta investigación se centra en analizar esta competencia en el alumnado que ingresa en la universidad. $Y$ es que desde la implementación de Internet, como método de acceso a la información, comenzaba a indicarse la urgente necesidad de formar a las nuevas generaciones en estas habilidades. Hace más de una década Tejedor (2008) advertía que las personas que no adquiriesen las competencias digitales, en unos años serían consideradas como analfabetas y tendrían una posición de desventajada en la sociedad (citado en Gutiérrez y Serrano, 2018, p. 78). Es por ello, que se pretende conocer si al alumnado universitario de nuevo ingreso ha adquirido esta competencia en los ciclos formativos precedentes al universitario.

Estudios nacionales e internacionales están abordando "como la tecnología debe ser incluida como núcleo fundamental de estudio en los niveles primarios y secundarios" (Cabero, 2006, p.159). Y el elevado número de investigaciones centradas en detectar las habilidades que tiene el estudiantado en las instituciones de educación superior para acceder y localizar información en Internet, indican la importancia dada a nivel mundial. Tómese como referencia los realizados en la Universidad Estatal de Lima Metropolitana (Sausuure y Portilla, 2017); la Universidad Católica de Santo Toribio de Mogrovejo en Chiclayo de Perú (Rumiche y Chunga, 2019), o el Instituto de Ciencias Humanas y de la Información de la Universidad Federal de Rio Grande (Bermúdez, Braz y Serrano, 2015).

A nivel europeo, destacables son las investigaciones realizadas a estudiantes de psicología alemanes (Leichner, Peter, Mayer y Krampen, 2013); doctorandos de la Universidad de Varsovia en Polonia y de la Universidad de Lille en Francia (Wiorogórska, 2014); estudiantado de la Universidad de Parma (Vezzosi, 2009); o del Instituto de tecnología de la Universidad de Descartes de París (Boustany, 2013). Si bien, otros enfoques significativos lo conforman las investigaciones que analizan la presencia de esta competencia en los planes de estudios, como el abordado en la Universidad de Zadar (Rubinic, Stričević y Juric, 2013); o los que exponen los resultados obtenidos de los cursos impartidos sobre alfabetización informacional (Špiranec y Pejova, 2010) y los métodos usados para impartir estas competencias (Spivakovska, Osipova,Vinnik y Tarasich, 2014); junto a los que informan sobre 
De Vicente Domínguez, A. M., Carballeda Camacho, M. R. y Cestino González, E. Análisis de las competencias mediáticas del alumnado que ingresa en la universidad: un estudio de caso en estudiantes de comunicación

proyectos creados por la Unión Europea, como el impulsado dentro del sector programático Erasmus + en forma de Massive Open Online Course (MOOC) coordinado por la Universidad de Graz denominado "Information Literacy Online" (Dreisiebner y Mandl, 2017).

En las universidades españolas, que es donde se enmarca esta investigación, los estudios se han realizado principalmente en las Facultades de Educación, es decir, se han centrado en las capacidades y habilidades de los futuros maestros. Se ha analizado el nivel de conocimientos, uso y actitudes hacia las TIC por parte del alumnado de magisterio de Albacete (especialidad en educación infantil y primaria pertenecientes al segundo curso) en la Universidad de Castilla la Mancha (del Valle, Hernández Bravol; Hernández Bravo y Cózar, 2011); investigado, junto a otras variables, las competencias en navegación, búsqueda y filtrado de información en el alumnado del grado de educación infantil y primaria de la Universidad Internacional de Valencia (Moreno, Garbada, y Rodríguez, 2018); así como, indagado en las predisposiciones y comportamientos informativos de los jóvenes universitarios respecto a la actividad de búsqueda en Internet de la Facultad de Educación de la Universidad de Salamanca (Hernández y González, 2010); o en los procesos de búsqueda, acceso y selección de información digital de los universitarios de magisterio de educación infantil y primaria de la ISEN Cartagena, un centro adscrito a la universidad de Murcia, (Gutiérrez y Serrano, 2018); o en los estudiantes de magisterio de la Universidad de Jaén (Molero, 2005).

Unas competencias que también se han investigado en los universitarios que estudian traducción e interpretación en la Universitat Autónoma de Barcelona con el fin de conocer sus hábitos de consulta y fines académicos (Cid y Perpinyá, 2015); o en los estudiantes de último año de carrera de Alicante (Laguna, 2013) para dar a conocer como se documentan para realizar las tareas académicas; así como, en el alumnado de enfermería del campus Terres de L'Ebre en la Universitat Rovira i Virgili para buscar y seleccionar información académica en Internet (Camacho, 2011); o bien, del estudiantado de la Universidad de las Islas Baleares (Comas, Pastor y Morey, 2011); y realizado diagnósticos sobre las competencias informacionales basadas en la percepción por parte de los estudiantes de la Universidad de Granada de las ramas del conocimiento en Artes y Humanidades, Ciencias, Ciencias de la Salud, Ciencias Sociales y Jurídicas, e Ingeniería y Arquitectura, (Pinto y Guerrero, 2011).

Otros estudios se han centrado en analizar estas competencias en los universitarios matriculados en la Facultad de Comunicación Audiovisual de la Universidad de Mondragon Unibertsitatea (Egaña, Bidegain y Zuberogoitia, 2013), y en averiguar la opinión de los estudiantes de la titulación de Comunicación Audiovisual de la Universidad de Granada sobre sus propias competencias y habilidades en el manejo y uso de la información, (Puertas y Pinto, 2010). 
De Vicente Domínguez, A. M., Carballeda Camacho, M. R. y Cestino González, E. Análisis de las competencias mediáticas del alumnado que ingresa en la universidad: un estudio de caso en estudiantes de comunicación

Ahora ampliamos esta línea de investigación analizando las competencias en búsqueda de información en Internet que trae el alumnado que ingresa en el grado de Publicidad y Relaciones Públicas de la Universidad de Málaga. Se analiza el tipo de recursos (impresos o digitales) que los estudiantes usan para sus trabajos académicos, cuáles son los principales buscadores que utilizan y los conocimientos que tienen sobre sus herramientas y servicios. Y es que el Real Decreto 1631/20064, de 29 de diciembre establecía que una de las enseñanzas mínimas correspondientes a la Educación Secundaría sería adquirir habilidades para acceder a la información incluyendo las tecnologías de la información como elemento esencial para informarse, aprender y comunicarse. Motivo por el que se pretende conocer si estas nuevas generaciones, traen estas habilidades adquiridas. Un estudio que se centra en los buscadores, pues como ya advertían Nachmias y Gilad (2002) son diversos los investigadores que recomiendan más estudios sobre el comportamiento de los usuarios frente a los buscadores. Unos datos sobre su adquisición que son "un reflejo de la medida en que los sistemas educativos están o no a la altura de las nuevas sociedades de la información" (Waheed, 2009, p, 6).

\section{OBJETIVOS Y METODOLOGÍA}

Los objetivos que se plantean en el estudio son: investigar si el alumnado usa recursos digitales o impresos para la elaboración de sus trabajos académicos; identificar los recursos impresos y digitales que utilizan para documentarse; detectar cuál es su principal buscador para localizar información; identificar si conocen los comandos $\mathrm{u}$ operadores y los indicadores booleanos como estrategias de búsqueda; valorar si saben que son los metabuscadores y buscadores especializados; e indicar cuáles son los principales metabuscadores y buscadores especializados que conocen. Y para la metodología se ha basado en los siguientes fases:

Revisisón bibliográfica en bases de datos nacionales e internacionales (dialnet, Bon, Google scholar, Teseo, Scopus, Wos, SAGE Journals on line, Taylor \& Francis) para conocer las investigacciones precedentes y comprobar que este estudio no ha sido realizado con anteroridad. Se emplearon los descriptores "alfabetización informacional", "alfabetización mediática", "búsqueda en Internet", y "competencias informacionales" combinándolos a través del conector booleano AND con las palabras claves "universidad", "instituciones de educación superior", "estudiantes" y "comunicación".

Selección de la muestra: se selecciona todo el alumnado de una materia obligatoria de primer curso del plan de estudios del grado de Publicidad y Relaciones Públicas de la Universidad de Málaga (curso 2020-2021). En concreto, se aborda en el estudiantado de la asignatura Fundamentos del Periodismo tanto del turno de mañana como de la tarde $(n=130)$. Y siguiendo la fórmula de Sierra Bravo (2007) para poblaciones finitas se analiza la población total del alumnado matriculado $(n=130)$, cuya edad media es de 18, 6 años. 
De Vicente Domínguez, A. M., Carballeda Camacho, M. R. y Cestino González, E. Análisis de las competencias mediáticas del alumnado que ingresa en la universidad: un estudio de caso en estudiantes de comunicación

Instrumento para la recogida de datos: se toma como referencia la metodología usada en estudios precedentes basada en obtener datos cuantitativos mediante un cuestionario (Cid y Perpinya, 2015; Álvarez y Gisbert, 2015; Egaña, Bidegain y Zubero, 2013; Moreno, Gabarda y Rodríguez, 2018). Asimismo, el estudio de AcostaSilva (2017) indica que esta es la principal metodología usada en las investigaciones publicadas en español e inglés que abordan investigaciones sobre el campo objeto de estudio, pues en el $75 \%$ de la muestra analizada el principal instrumento de recogida de datos eran los cuestionarios.

Un cuestionario que está conformado por siete items agrupados en tres variables variables: tipología (ítems 1 y 2); buscador principal (ítems 3); competencias de búsqueda (ítems 4, 5, 6 y 7). Unos códigos que se basan en un diseño similar de estudios precedentes.

Tabla 1: Ítems del cuestionario

\begin{tabular}{|l|}
\hline Tipología \\
\hline 1. ¿Usas recursos digitales o impresos para realizar un trabajo académico? \\
2. Identifica los recursos impresos y digitales \\
\hline Buscador principal \\
\hline 3. ¿Cuál es su principal buscador para localizar información? \\
\hline Competencias de búsqueda \\
\hline 4. ¿Qué comandos u operadores conoces para localizar información en tu buscador \\
principal? \\
5. ¿Sabes que son los indicadores booleanos? ¿Cuáles conoces? \\
6. ¿Qué metabuscadores conoces? ¿Cuáles son? \\
7. ¿Qué directorios específicos conoces? ¿Cuáles son? \\
\hline
\end{tabular}

Fuente: Elaboración propia.

Las preguntas son abiertas para evitar que el alumnado escogiera entre un conjunto finito de alternativas, haciendo uso de una las ventajas de esta modalidad indicaba por Pope (2012) que es la "recolección de información espontánea" (citado en Rincón, 2014, p. 141) y también porque son las usadas cuando se trata de explorar y profundizar en un tema.

El cuestionario se subió al campus virtual de la materia y el alumnado procedió a cumplimentarlo en horario de clase con un tiempo estimado de respuesta de 10 minutos sin producirse ninguna incidencia. Los datos no están disponibles en Internet y son confidenciales. De los 130 alumnos que conforman la población total de la muestra respondieron al cuestionario 108 alumnos, lo que implica un nivel de confianza del $95 \%$ y un margen de error del $4 \%$. La recogida de datos se realizó el 9 de abril de 2021.

Técnica de recolección de datos: la técnica de análisis para analizar la data se realiza agrupando las respuestas en categorías, otorgándoles un código con su correspondiente asignación numérica para poder ser tabuladas y codificadas. 
De Vicente Domínguez, A. M., Carballeda Camacho, M. R. y Cestino González, E. Análisis de las competencias mediáticas del alumnado que ingresa en la universidad: un estudio de caso en estudiantes de comunicación

\section{RESULTADOS Y DISCUSIÓN}

Los resultados indican un elevado uso de Internet para realizar las tareas académicas, pues un $48 \%$ usan exclusivamente material on line y un $49 \%$ lo combinan con material impreso, no optando nunca por usar sólo material impreso. Estos datos implican que Internet es su principal recurso documental. Una tendencia ya implementada desde hace décadas. Egaña, Bidegain y Zuberogoitia (2013) indicaban: "Internet es desde hace ya algunos años la principal fuente de información de los estudiantes universitarios (British Library; JICS, 2008; Fuentes Agustí; Monereo, 2008; Benítez de Vendrell, 2007; Sureda; Comas, 2006; Nazim; Saraf, 2006; OCLC, 2005)" (p.2). Unos resultados que también corroboran los aportados por Comas, Sureda, Pastor y Morey (2011).

Este elevado uso se considera debido a que los nativos digitales "manejan las tecnologías de forma normalizada en casi todos los aspectos de su vida" (Larrañaga y Cubero, 2019, p. 52) y por tanto, también lo aplican para sus tareas académicas. Si bien, otras teorías indican que se debe a que los estudiantes son "conscientes de que en la web encontrarán todo lo que necesitan para ejecutar sus tareas" (Balaguera y Villegas, 2012, p. 161).

Se destaca, no obstante, que aunque la búsqueda de información para tareas académicas sea principalmente mediante recursos digitales, el acceso a la cultura impresa sigue formando parte del proceso de búsqueda en uno de cada dos universitarios, pues el $49 \%$ de la muestra analizada combina los recursos digitales con los impresos. Gutiérrez-Porlán, Román-García y Sánchez-Vera (2018) consideran que cuando "las tareas se vuelven más complejas y requieren mayor implicación, el empleo de Internet es más bajo (citado en Elche y Yubero, 2020, p. 53). Una combinación que otros investigadores exponen que se produce porque los jóvenes universitarios tienen acceso a diferentes medios de información digitales y soportes tecnológicos para la lectura que se complementa con la lectura de fuentes impresas tradicionales (Chávez, Flores, Ordoñez y Sánchez, 2020). Si bien, esa combinación de formatos se genera, según Kitamura (2013), dependiendo del dispositivo de uso, pues desvela que el uso de Internet a través de teléfonos móviles tiene efectos complementarios en la adquisición de información de los medios tradicionales, mientras que el uso de Internet a través de computadoras personales no los tiene.

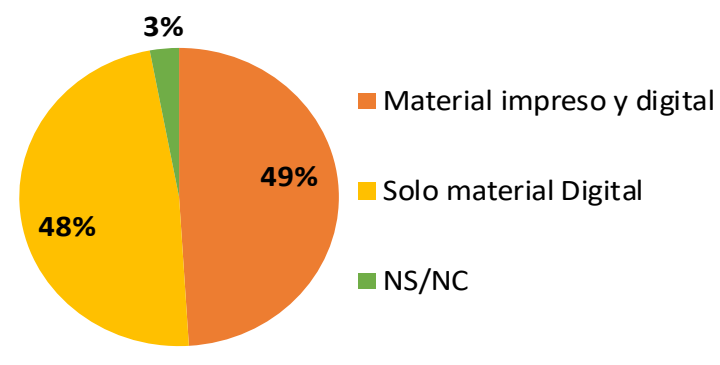

Figura 1: Tipo de recursos usados para las tareas académicas

Fuente: Elaboración propia. 
De Vicente Domínguez, A. M., Carballeda Camacho, M. R. y Cestino González, E. Análisis de las competencias mediáticas del alumnado que ingresa en la universidad: un estudio de caso en estudiantes de comunicación

Cuando recurren a documentación impresa, los resultados muestran que consultan principalmente, por orden decreciente: libros (37\%), apuntes $(15 \%)$, periódicos $(12 \%)$, revistas $(11 \%)$, enciclopedias $(10 \%)$, y diccionarios $(8 \%)$. Mientras que los principales recursos digitales que usan son por orden decreciente: páginas webs $(20 \%)$, redes sociales $(12 \%)$, periódicos $(10 \%)$, blog $(9 \%)$, videos $(8 \%)$, revistas digitales $(6 \%)$, artículos (5\%), presentaciones (5\%), Wikipedia (4\%), libros (3\%), podcast $(2 \%)$, trabajos académicos $(2 \%)$, apuntes $(1 \%)$, tesis $(1 \%)$ y TFG $(1 \%)$.

Medina (2020) considera como "Internet y las fuentes web parecen haber relegado a los libros, ya sean impresos o electrónicos (...) a un papel más que secundario" (p. 88). Y estos datos revelan cómo los libros siguen siendo el principal recurso, cuando acceden al formato impreso para documentarse, pero que cuando recurren el entorno digital tienen preferencia por las páginas web y las redes sociales. Bonilla, Diego y Lena (2018) también obtienen en un estudio realizado a más de 200 universitarios españoles que estos emplean las redes sociales "a menudo para trabajar de forma colaborativa y ayudarse en las tareas académicas" (p. 324) resaltando que los estudiantes no son productores de contenidos, pero sí consumidores mediáticos.

Significativo es que un 3\% de las respuestas han sido erróneas pues el alumnado ha indicado como recurso digital para localizar información el móvil, la televisión, el ordenador o portátil y la Tablet, cuando son los instrumentos para acceder a Internet.

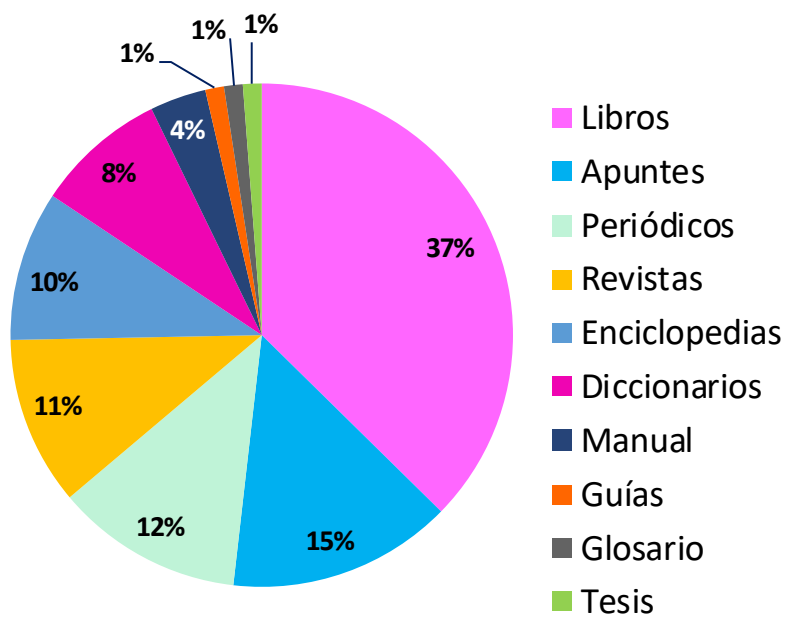

Figura 2: Recursos impresos utilizados para los trabajos académicos

Fuente: Elaboración propia. 
De Vicente Domínguez, A. M., Carballeda Camacho, M. R. y Cestino González, E. Análisis de las competencias mediáticas del alumnado que ingresa en la universidad: un estudio de caso en estudiantes de comunicación

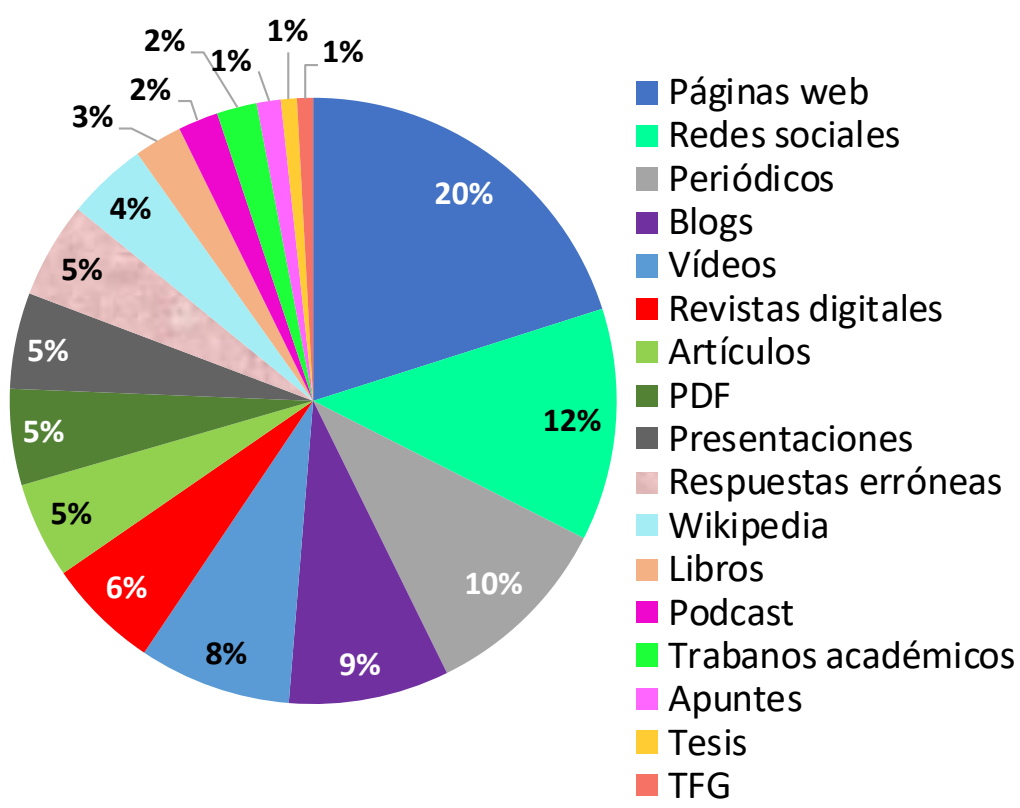

Figura 3: Recursos digitales utilizados para las tareas académicas

Fuente: Elaboración propia

Los datos obtenidos sobre los buscadores desvelan que un $84 \%$ de la muestra usa como principal buscador Google. Si bien, un 9\% de la muestra objeto de estudio tienen preferencia por otros buscadores que por orden decreciente son: Google académico (5\%), Bing (2\%) y Ecosia (2\%). El 7\% restante indica no saber cuál es su principal motor de búsqueda o no contestan. Unos datos que indican una elevada preferencia por Google y que coindice con los obtenidos en estudios precedentes (Sureda, Comas y Urbina, 2006; Hernández y González, 2011; Comas, Pastor y Morey 2011; Egaña, Bidegain y Zuberohoitia, 2013; Cid y Perpinyá, 2015). De hecho, como indican Santovenía, Andalia, Ochandarena y Brito (2007) "la entrada de google en diversos diccionarios refleja la popularidad del buscador hasta el punto que el término googlear se convirtió para millones de internautas en sinónimo de búsqueda de información" (p. 3).

Se destaca que el alumnado presenta deficiencias en un 6\% de conceptualización, pues al preguntarles algunos confunden navegadores con buscadores: exponen que su principal buscador es Safari, Firefox, Opera, Chrome o Edge, siendo éstos navegadores. 
De Vicente Domínguez, A. M., Carballeda Camacho, M. R. y Cestino González, E. Análisis de las competencias mediáticas del alumnado que ingresa en la universidad: un estudio de caso en estudiantes de comunicación

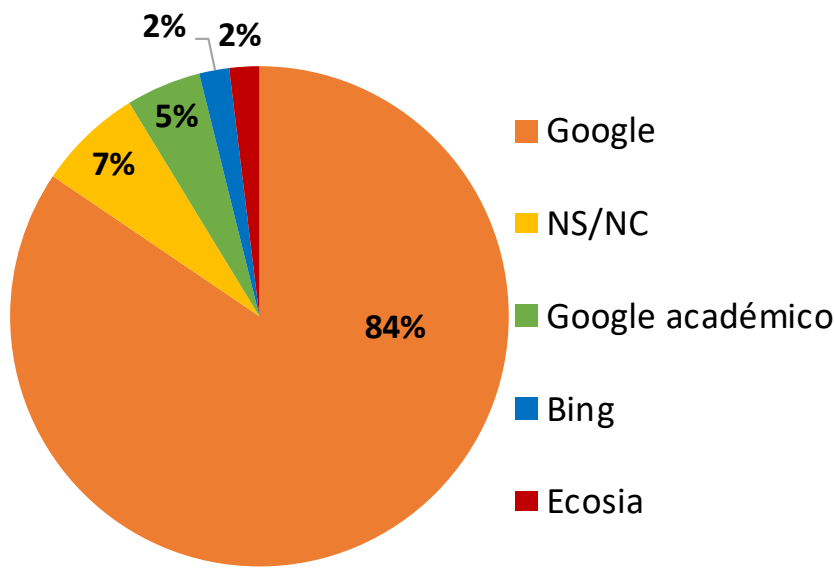

Figura 4: Principal buscador

Fuente: Elaboración propia.

En este contexto, González Rivera y Santana (2008) advierten que resulta cada vez más alarmante "la preferencia alcanzada por el buscador Google, un motor que, aun cuando presenta un enorme potencial, debe explotarse con mucho cuidado, sobre todo por 'manos inexpertas', teniendo en cuenta las ostensibles diferencias de calidad que presentan los recursos recuperados por medio de la búsqueda en este motor" (p. 3). Y es que como desvelan los resultados, el alumnado objeto de la muestra, usa este buscador, pero no tienen competencias para localizar correctamente la información.

Los resultados indican que un $89 \%$ no conoce los indicadores booleanos frente un $11 \%$ que indican conocernos. Si bien, del 11\% que saben para que sirven sólo un 36\% los usa frente a un 55\% que nunca los insertan en sus procesos de búsqueda y un $6 \%$ que indican utilizarlos a veces. Es así, que el alumnado desconoce cómo establecer las relaciones lógicas entre los términos de búsqueda, porque principalmente no conocen la existencia de los operadores booleanos: OR, AND y NOT. Este rechazo e ignorancia del uso de los indicadores booleanos en los estudiantes universitarios coinciden con las investigaciones de Gutiérrez y Serrano (2018).

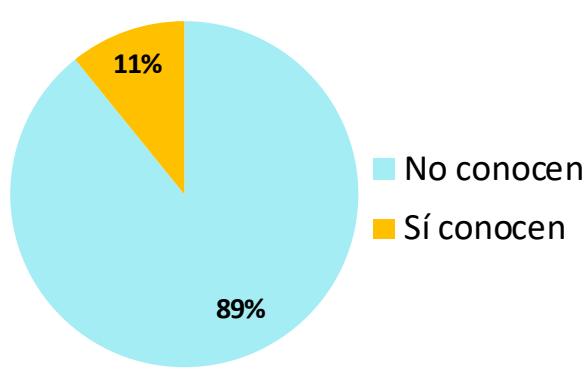

Figura 5: Conocimiento de los operadores booleanos

Fuente: Elaboración propia. 
De Vicente Domínguez, A. M., Carballeda Camacho, M. R. y Cestino González, E. Análisis de las competencias mediáticas del alumnado que ingresa en la universidad: un estudio de caso en estudiantes de comunicación

Los datos también desvelan que no tienen una formación informacional del uso de los comandos y los operados de Google para localizar los contenidos en la ingente cantidad de datos que hay en Internet. Si bien, un 51\% indica que los conocen frente a un $49 \%$ que desconocen su existencia, su conocimiento es escaso entre quienes lo identifican pues por orden decreciente: un $52 \%$ tan sólo especifica uno, un $20 \%$ tres, un $17 \%$ dos, un $5 \%$ cuatro comandos, un $3 \%$ seis y otro $3 \%$ conoce siete comandos.

Se destaca también una disfunción de conceptualización al preguntarles por los comandos porque algunos responden que son el uso de palabras claves, las cookies o Chrome, junto a otros.

Y atendiendo a otras competencias de búsqueda, los datos indican que un $78 \%$ no saben que existen buscadores especializados frente a un $22 \%$ que sí indican conocerlos, aunque como ha sucedido con los comandos u operadores de Google son escasos los identificados pues del 22\% que indican conocerlos: un $74 \%$ sólo expone uno, un $14 \%$ dos, un $6 \%$ tres buscadores especializados, un $5 \%$ cuatro y $1 \%$ cinco. En concreto, el más representado con un $71 \%$ es Google académico. Y con una muy escasa representación exponen: Chemedia (6\%); Dialnet (4\%); Educaweb (4\%), Sciencie Research (2\%) o Scielo (2\%). Así, como ya desvelaban Cid y Perpiñan (2015) entre los recursos desconocidos por los jóvenes universitarios se incluirían buscadores especializados, directorios y bases de datos bibliográficas especializadas.

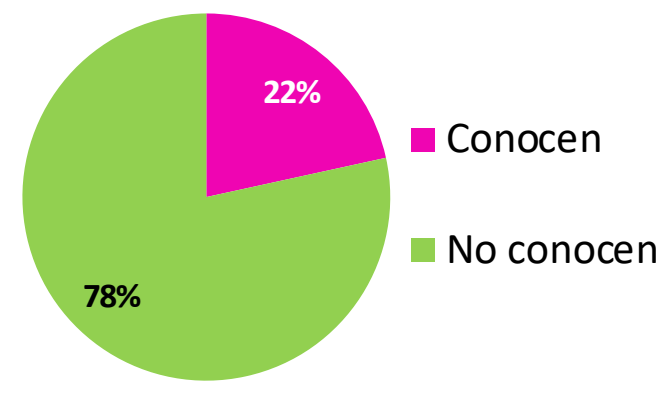

Figura 6: Conocimiento de buscadores especializados

Fuente: Elaboración propia. 
De Vicente Domínguez, A. M., Carballeda Camacho, M. R. y Cestino González, E. Análisis de las competencias mediáticas del alumnado que ingresa en la universidad: un estudio de caso en estudiantes de comunicación

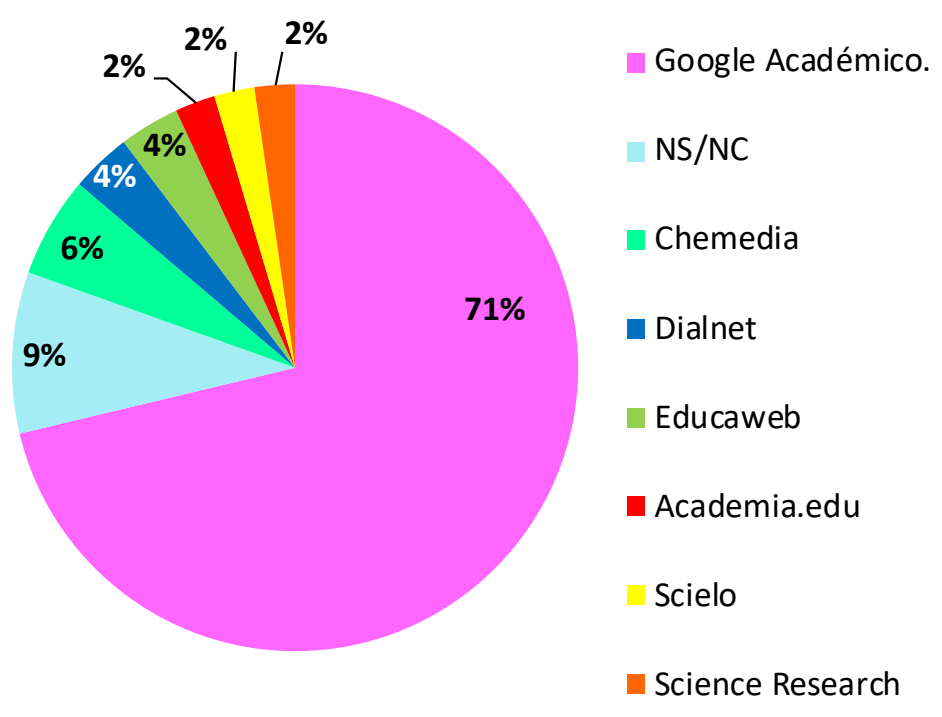

Figura 7: Identificación de los buscadores especializados Fuente: Elaboración propia.

Respecto a los metabuscadores, los datos desvelan que un $63 \%$ no conoce ninguno, frente a un 37\% que sí indica conocerlos, siendo estos por orden decreciente: Dogpile (26\%); Onessek (13\%); Metacrawer (5\%); Duckdukgo (2\%) y Looksmart (2\%). Si bien, se destaca que $52 \%$ de las respuestas sobre metabuscadores conocidos son erróneas: así, por ejemplo, un $24 \%$ considera que Yahoo! es un metabuscador, cuando se trata de un motor de búsqueda. O un $2 \%$ cree que el correo de Gmail es un metabuscador. Un desconocimiento que también desvelan Hernández y Fuentes, 2011.

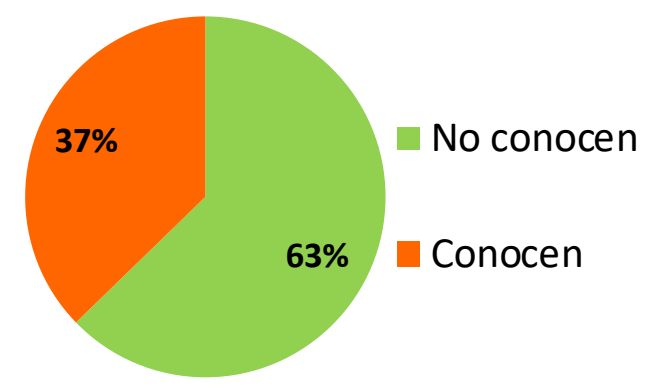

Figura 8: Conocimiento de los Metabuscadores Fuente: Elaboración propia. 
De Vicente Domínguez, A. M., Carballeda Camacho, M. R. y Cestino González, E. Análisis de las competencias mediáticas del alumnado que ingresa en la universidad: un estudio de caso en estudiantes de comunicación

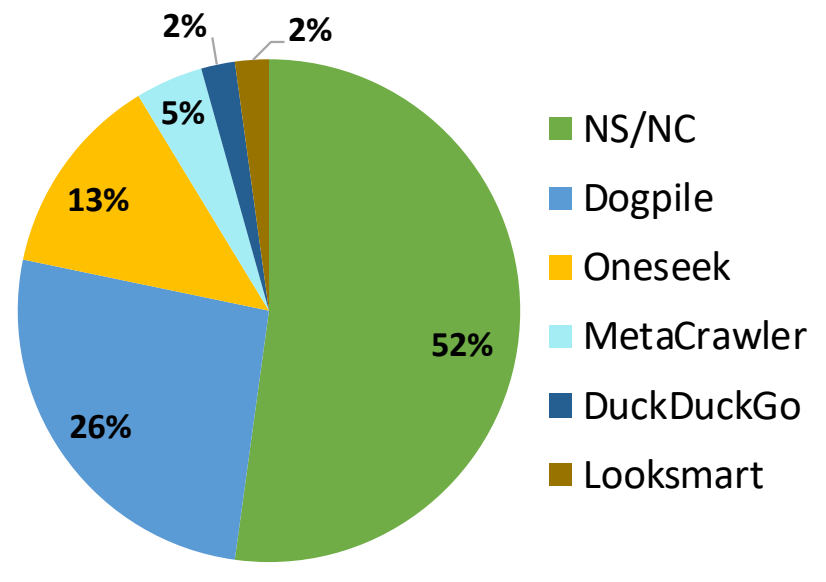

Figura 9: Identificación de los metabuscadores

Fuente: Elaboración propia.

Cabra Torres y Marciales-Vivas (2009) desvelaban la existencia de una “insuficiente evidencia empírica para caracterizar los nativos digitales como usuarios hábiles en el empleo de diversas tecnologías" (p. 330). Y este estudio corrobora que tienen un dominio instrumental de los dispositivos pero que efectivamente eso no implica dominar las habilidades y competencias para gestionar el acceso de la información en Internet para los trabajos académicos. Así como, tampoco implica dominar las tecnologías con nacer en un entorno tecnológico. Unos datos que coinciden con el estudio de Castellano, Sánchez y Calderero (2017): los alumnos universitarios estudiados no comparten los rasgos que se esperan de un nativo digital, en especial en lo que se refiere consumir cultura a través de Internet. (citado en Henríquez, Andrade y Moreno, 2018, p. 61)

Gutierrez y Serrano (2018) también lo corroboran y destacan que otras investigaciones (Egaña, Bidegain y Zuberogoitia, 2013; Egaña, Zuberogoitia, Pavón y Brazo, 2012; Fuentes y Monereo, 2008; Gutiérrez y Serrano, 2016; Hernández Serrano, 2009; Hernández y Fuentes, 2011; Laguna, 2013 y Valadez, Páez y Zapata, 2005) (p. 86 también indican que parece existir evidencias de que los estudiantes universitarios no siempre son lo deseablemente competentes buscando y accediendo a la información digital.

\section{CONCLUSIONES}

La contribución de este estudio a las implicaciones teóricas aportadas sobre el grado de habilidad de las generaciones nativas digitales para buscar información en Internet es afianzar la postura de que los nativos digitales que están cursando estudios universitarios no tienen competencias para buscar información en Internet. No han traído estas competencias adquiridas de los ciclos formativos precedentes, o al menos, los de la muestra analizada no las tienen adquiridas. Es así, que se 
De Vicente Domínguez, A. M., Carballeda Camacho, M. R. y Cestino González, E. Análisis de las competencias mediáticas del alumnado que ingresa en la universidad: un estudio de caso en estudiantes de comunicación

concluye con la urgente necesidad, ya advertida desde décadas atrás, de fomentar estrategias educativas que permitan la gestión de estas habilidades y actitudes, porque los estudiantes presentan una alfabetización instrumental pero no informacional.

El estado actual de los procesos de búsquedas del alumnado analizado inclina la balanza hacia las teorías que afirman que se está generando una nueva cultura informativa para acceder a la documentación con la que realizar los trabajos académicos. Si bien, esa cultura se basa en el elevado uso de internet para realizar las búsquedas, se concluye que la cultura impresa sigue siendo un recurso también utilizado aunque siempre combinado con los recursos digitales. Es así, que en la gestión del conocimiento de la cultura digital, no debe obviarse la continua formación de la cultura letrada o impresa, con el fin de que se sigan complementado lo tradicional con los avances tecnológicos.

Este trabajo aporta a la literatura existente una radiografía de las disfunciones presentadas, cuya identificación se considera aptas para el desarrollo de programas formativos que permitan paliarlas. En concreto, es necesario formarles en indicadores booleanos, metabuscadores, buscadores especializados, comandos $u$ operadores de Google. Por tanto, estas carencias deben resolverse también mediante cursos específicos, o bien, de forma transversal en cada una de las materias docentes.

Se concluye que también presentan otras carencias como confundir recurso digital con los instrumentos que permiten acceder a Internet (ordenador, televisión o móvil), no diferenciar entre navegadores o buscadores o creer que los comandos son las cookies. Lo que hace fundamental formar también al alumnado en estas conceptualizaciones.

Asimismo, dado que tienden a usar Internet para realizar búsquedas documentales se recomienda que los docentes, en cada una de sus especialidades o materias que impartan, aporten al alumnado páginas webs de interés para su consulta, así como perfiles sociales que consideren de utilidad porque tienden a recurrir a estas fuentes principalmente para localizar contenidos. Así como, todos aquellos recursos digitales que los docentes consideren de interés para mejorar estas competencias. Es así, que es necesario que la educación se adapte a las nuevas tendencias, sin dejar de lado la cultura impresa.

Estudios futuros analizaran estas competencias en muestras más grandes que permitan obtener resultados generales del total de la población universitaria española que ingresa en la universidad. Si bien, una de las delimitaciones de este estudio es que se trata de un grupo específico, se aporta unos datos indicativos para calibrar si se está implementando las competencias informacionales a estas generaciones para acceder a la información especializada y desarrollar una documentación significante. 
De Vicente Domínguez, A. M., Carballeda Camacho, M. R. y Cestino González, E. Análisis de las competencias mediáticas del alumnado que ingresa en la universidad: un estudio de caso en estudiantes de comunicación

\section{REFERENCIAS}

Acosta-Silva, D. (2017). Tras las competencias de los nativos digitales: avances de una metasíntesis. Revista Latinoamericana de Ciencias Sociales, 15(1), 471-489.

Balaguera, E., y Villegas, M.M. (2012). Criterios para tomar la información suministrada por la web: Una perspectiva con estudiantes universitarios. Paradígma, 33(2), 159-172.

Bonilla-del-Río, M., Diego-Mantecón, J. M., y Lena-Acebo, F. J. (2018). Estudiantes Universitarios: prosumidores de recursos digitales y mediáticos en la era de Internet. Aula Abierta, 47(3), 319-326. https://doi.org/10.17811/rifie.47.3.2018.319$\underline{326}$

Boustany J. (2013). Information Literacy Skills of Students at Paris Descartes University. En Kurbanoğlu S., Grassian E., Mizrachi D., Catts R., Špiranec S. (eds) Worldwide Commonalities and Challenges in Information Literacy Research and Practice. Communications in Computer and Information Science, vol 397. Springer, Cham. https://doi.org/10.1007/978-3-319-03919-0_79

Cabero, J. (2006). Capacidades tecnológicas de las TICS por los estudiantes. Enseñanza, 24, 159-175.

Castellanos, A., Sánchez, C. y Calderero, J. F. (2017). Nuevos modelos tecnopedagógicos. Competencia digital de los alumnos universitarios. Revista Electrónica de Investigación Educativa, 19(1), 1-9.

Chávez-Márquez, I.L, Flores-Morales, C.R., Ordóñez Parada, A.I. y Sánchez Acosta, L.R. (2020). Nativos digitales: internet y su relación con la lectura en estudiantes universitarios. Apertura (Guadalajara, Jal.), 12(2), 94-107. https://doi.org/10.32870/ap.v12n2.1876

Coll, C. (2005). Lectura y alfabetismo en la sociedad de la información. UOC Papers,1, 4-10. https://n9.cl/emzk

Comas, R., Sureda, J., Pastor, M., y Morey, M. (2011). La búsqueda de información con fines académicos entre el alumnado universitario. Revista Española De Documentación Científica, 34(1), 44-64. https://doi.org/10.3989/redc.2011.1.769

Cid-Leal, P y Perpinyà-Morera, R. (2015). Competencia informacional en Traducción: análisis de los hábitos de los estudiantes universitarios en la consulta y uso de fuentes de información. Textos universitarios de biblioteconomía y documentación,34 https://dx.doi.org/10.1344/BiD2015.34.11 
De Vicente Domínguez, A. M., Carballeda Camacho, M. R. y Cestino González, E. Análisis de las competencias mediáticas del alumnado que ingresa en la universidad: un estudio de caso en estudiantes de comunicación

De Moya-Martínez, M.V.; Hernández-Bravo, J.R.; Hernández-Bravo, J.A y CózarGutiérrez, R. (2011). Análisis de los estilos de aprendizaje y las TIC en la formación personal del alumnado universitario a través del cuestionario REATIC. Revista de Investigación Educativa, 29(1),

http://revistas.um.es/rie/article/view/110481/126972.pdf

Dreisiebner, S. \& Mandl, T. (2017). Entwicklung und Evaluierung einer Plattform zur Vermittlung von Informationskompetenz: Vorstellung des von der Europäischen Union geförderten Projekts „Information Literacy Online“. Bibliotheksdienst, 51(9), 763-770. https://doi.org/10.1515/bd-2017-0083

Egaña, T., Zuberogoitia, A., Pavón, A., y Brazo, L. (2012). ¿Cómo evalúan la información de internet los estudiantes universitarios? Lo que dicen los estudiantes y sus profesores. EDUTEC. Revista Electrónica de Tecnología Educativa, 42. http://dx.doi.org/10.21556/edutec.2012.42.347

Egaña, T., Bidegain, E. y Zuberogoitia, A. (2013). ¿Cómo buscan información académica en internet los estudiantes universitarios? Lo que dicen los estudiantes y sus profesores. EDUTEC. Revista Electrónica de Tecnología Educativa, 43, 1-15. http://dx.doi.org/10.21556/edutec.2013.43.332

Figueroa-Portilla, C.S. (2016). El uso del smartphone como herramienta para la búsqueda de información en los estudiantes de pregrado de educación de una universidad de Lima Metropolitana. Educación, 25(49), 29-44. https://dx.doi.org/10.18800/educacion.201602.002

Fuentes-Agustí, M. y Monereo-Font, C. (2008). Cómo buscan información en Internet los adolescentes. Revista Investigación en la Escuela, 64, 45-58. http://www.investigacionenlaescuela.es/articulos/64/R64_4.pdf

García-Martínez, M. (2011). Competencia informacional de los estudiantes de enfermería del campus Terres de l'Ebre para buscar y seleccionar información académica en internet. [Tesis doctoral, Universitat Rovira i Virgili] http://hdl.handle.net/10803/8952

Gisbert, M. y Esteve, F. (2011). Digital Learners: la competencia digital de los estudiantes universitarios. La cuestión universitaria, 7, 48-59. http://polired.upm.es/index.php/lacuestionuniversitaria/article/view/3359

Godinho, N. B., Gonçalves, R. B., \& Almeida, A. S. de. (2015). Competências digitais e informacionais no ensino superior: um estudo com acadêmicos na Universidade Federal do Rio Grande - FURG. RDBCI: Revista Digital De Biblioteconomia E Ciência Da Informação, 13(2), 437-454. https://doi.org/10.20396/rdbci.v13i2.8635591

Gómez-Hernández, J.A. (2002). La alfabetización informacional como servicio de las bibliotecas. Referencias, 7(1), 5-14. 
De Vicente Domínguez, A. M., Carballeda Camacho, M. R. y Cestino González, E. Análisis de las competencias mediáticas del alumnado que ingresa en la universidad: un estudio de caso en estudiantes de comunicación

González Rivero, M.C. y Santana Arroyo, S. (2008). Comportamiento de los estudiantes de medicina en la búsqueda de información en Internet. Revista Cubana de Información en Ciencias de la Salud, 17(4).

Gutierrez-Gómez, J. A. y Serrano-Sánchez, J. L. (2018). Análisis de los procesos de búsqueda, acuse, selección de información digital en futuros maestros. Digital Education Review, 34, 76-90.

Gutiérrez-Porlán, I., Román-García, M. y Sánchez-Vera, MM. (2018). Estrategias para la comunicación y el trabajo colaborativo en red de los estudiantes universitarios. Comunicar 26(54), 91-100. http:/ / dx.doi.org/10.2155610.3916/C54-2018-09

Henríquez-Coronel, P.M., Andrade, A. y Moreno-Hernández, Y. (2018). Conductas de búsqueda de información en Internet en estudiantes universitarios de Comunicación Social en Ecuador Revista latina de sociología,8(1), 54-64

Hernández-Serrano, M.J. (2013). La búsqueda y selección de la información online: análisis de las acciones estratégicas de los estudiantes universitarios. Teoría de la Educación. Educación y Cultura en la Sociedad de la Información, 14(2), 85-106

Hernández-Serrano, M. J. y Fuentes-Agustí, M. (2011). Aprender a informarse en la red: ¿son los estudiantes eficientes buscando y seleccionando información? Teoría de la Educación: Educación y Cultura en la Sociedad de la Información,12(1), 47-78.

Hernández-Serrano, M. J., González-Sánchez, M., y Jones, B. (2011) La generación Google. Evolución en las predisposiciones y comportamientos informativos de los jóvenes. Revista Interuniversitaria de Pedagogía Social, 18, 41-56. http://dx.doi.org/10.7179/PSRI_2011.18.04

Johnston, B, \& Webber, S. (2003) Information Literacy in Higher Education: A review and case study, Studies in Higher Education, 28(3),335-352, https://doi.org/10.1080/03075070309295

Kitamura, S. (2013). The relationship between use of the internet and traditional information sources: An empirical study in Japan. SAGE Open, 1-9. https://doi.org/10.1177/2158244013489690

Laguna-Segovia, M. I. (2013). Estudio sobre el uso de Internet y sus aplicaciones en el alumnado de último año de carrera de la Universidad de Alicante. [Tesis Doctoral. Universidad de Alicante] https://dialnet.unirioja.es/servlet/tesis?codigo=55254

Larrañaga, M.E. y Yubero, S. (2019). La influencia del hábito lector en el empleo de internet: un estudio con jóvenes universitarios. Investigación bibliotecológica, 33(79), 51-66. https://doi.org/10.22201/iibi.24488321xe.2019.79.57985 
De Vicente Domínguez, A. M., Carballeda Camacho, M. R. y Cestino González, E. Análisis de las competencias mediáticas del alumnado que ingresa en la universidad: un estudio de caso en estudiantes de comunicación

Leichner, N., Peter, J., Mayer A.K. \& Krampen, G. (2013). Assessing information literacy among German psychology students. Reference Services Review 41(4), 660674. https://doi.org/10.1108/RSR-11-2012-0076

Medina Calzada, S. (2020). La alfabetización informacional en el alumnado de Estudios Ingleses: un estudio de caso. Aula de Encuentro, volumen 22(1), 81-110. https://doi.org/10.17561/ae.v22n1.4

Moreno-Rodríguez, M.D., Gabarda-Méndez, V. y Rodríguez-Martín A.M (2018). Alfabetización informacional y competencia digital en estudiantes de magisterio. Revista de Currículum y Formación de Profesorado, 22(3), 253270. http://digibug.ugr.es/handle/10481/53393

Nachmias, R. \& Gilad, A. (2002) Needle in a Hyperstack, Journal of Research on Technology in Education, 34(4), 475-486, https://doi.org/10.1080/15391523.2002.10782362

Pinto-Molina, M. y Guerrero-Quesada, D.J. (2017). Cómo perciben las competencias informacionales los estudiantes universitarios españoles: un estudio de caso. Investigación bibliotecológica, 31(73), 213-23. http://dx.doi.org/10.22201/iibi.24488321xe.2017.73.57854

Puertas-Valdeiglesias, S. y Pinto-Molina, M. (2010). El aprendizaje por competencias transversales: la competencia informacional y comunicacional de los estudiantes de la titulación de Comunicación Audiovisual. Diálogos de la comunicación, 80.

Rubinić D., Stričević I. \& Juric M. (2013). Information Literacy Course - The Perception of Students and Professors: University of Zadar Case. En: Kurbanoğlu S., Grassian E., Mizrachi D., Catts R., Špiranec S. (eds) Worldwide Commonalities and Challenges in Information Literacy Research and Practice (pp.528-534). Communications in Computer and Information Science, vol 397. Springer, Cham. https://doi.org/10.1007/978-3-319-03919-0_71

Rumiche, R. y Chunga, G. (2019). Evaluación de competencias digitales de estudiantes de la Universidad Católica de Santa Toribio de Mogrovejo (Perú). International Journal of New Education, 2, 75-89.

Salmerón Silvera, J.L., Bueno Ávila, S. y Luna Huertas, P. (2002). Localización de información en motores de búsqueda en Internet: análisis de la efectividad. Economía Industrial, 346, 173-182.

Santovenia Díaz, J., Cañedo Andalia, R., Ochandarena Mestre, R.J. y Brito Núñez N.M. (2007). Google: una aproximación al gran buscador. Revista Cubana de Información en Ciencias de la Salud, 15(4). 
De Vicente Domínguez, A. M., Carballeda Camacho, M. R. y Cestino González, E. Análisis de las competencias mediáticas del alumnado que ingresa en la universidad: un estudio de caso en estudiantes de comunicación

Špiranec, S., \& Pejova, Z. (2010). Information literacy in South-East Europe: Formulating strategic initiatives, making reforms and introducing best practices. The International Information $\mathcal{E}$ Library Review, 42(2), 75-83. https:// doi.org/10.1016/j.iilr.2010.04.002.

Spivakovska E., Osipova N., Vinnik M. \& Tarasich Y. (2014) Information Competence of University Students in Ukraine: Development Status and Prospects. In: Ermolayev V., Mayr H., Nikitchenko M., Spivakovsky A., Zholtkevych G. (eds) Information and Communication Technologies in Education, Research, and Industrial Applications, (pp. 94-216). Communications in Computer and Information Science, vol 469. Springer, Cham. https://doi.org/10.1007/978-3-319-13206-8_10

Sureda J. y Comas, R. (2006). Internet como fuente de documentación academica entre estudiantes universitarios. Una aproximación a partir del alumnado de Educación Social de la Universitat de les Illes Balears (UIB). Palma de Mallorca: Xarxa Segura IB.

Tejedor, F. J. (2008). Investigación educativa: su desarrollo en el ámbito de la Tecnología Educativa. En A. García-Valcárcel (coord.), Investigación y tecnologías de la información y comunicación al servicio de la innovación educativa, 177-208. Salamanca, España: Universidad de Salamanca

Toledo-Sánchez MC y Maldonado-Radillo SA. (2015). Alfabetización informacional en instituciones de educación superior: diseño de un instrumento de medición. biblios.pitt.edu, 60, 14-31. http://doi.org/10.5195/biblios.2015.245

Vezzosi, M. (2009). Doctoral students' information behaviour: an exploratory study at the University of Parma (Italy)", New Library World, 110(1), 65-80. https://doi.org/10.1108/03074800910928595

Waheed, A. K (2009). Prólogo. En R. Catts y J. Lau (coords.) Hacia unos Indicadores de Alfabetización Informacional (pp. 6-7). UNESCO, Ministerio de Cultura, Gobierno de España.

Wiorogórska, Z. (2014). Information Literacy and Doctoral Students in France and Poland. A Comparative Study. Zagadnienia Informacji Naukowej. Studia Informacyjne, 1(103), 42-66. 
De Vicente Domínguez, A. M., Carballeda Camacho, M. R. y Cestino González, E. Análisis de las competencias mediáticas del alumnado que ingresa en la universidad: un estudio de caso en estudiantes de comunicación

\section{AUTOR/ES:}

Aida María De Vicente Domínguez: Doctora en Comunicación por la Universidad de Granada. Especializada en Comunicación Digital. Profesora de los grados de Periodismo, Comunicación Audiovisual y Publicidad de la Universidad de Málaga. Forma parte del equipo del Grupo Comunicar de Málaga (Colectivo andaluz de Comunicación y Educación) y del comité científico de la colección de innovación educativa. Vía Docendi en la Universidad de las Palmas de Gran Canarias. Ha coordinado actualmente el monográfico "Transformación e innovación educativa durante la crisis del COVID-19. Estilos y modelos de enseñanza y aprendizaje" en la revista Estilos de Aprendizaje.

Orcid ID: https:// orcid.org/0000-0002-8085-5097

Mireya Rocio Carballeda Camacho: Licenciada en Comunicación Audiovisual por la Universidad de Málaga. Doctoranda del programa Interuniversitario de Doctorado en Comunicación. Profesora de los grados de Periodismo y Comunicación Audiovisual de la Facultad de Ciencias de la Comunicación de la Universidad de Málaga. Miembro del grupo de investigación sej435: contenidos audiovisuales avanzados (comunicación).

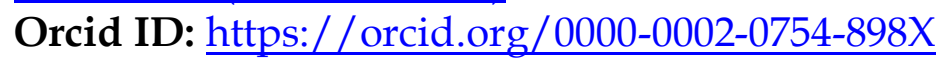

Estefanía Cestino González: Profesora del Departamento en Comunicación Audiovisual y Publicidad de la Universidad de Málaga. Licenciada en Comunicación Audiovisual. Diplomada en Logopedia. Maestra de Audición y Lenguaje.

Orcid ID: https:// orcid.org/0000-0002-2436-8665 